LETTER TO THE EDITOR

\title{
Polynomial solutions to the WDVV equations in four dimensions
}

\author{
R Martini and G F Post \\ Faculty of Applied Mathematics, University Twente, PO Box 217, 7500 AE Enschede, \\ The Netherlands
}

Received 30 December 1996

\begin{abstract}
All polynomial solutions of the WDVV equations for the case $n=4$ are determined. We find all five solutions predicted by Dubrovin, namely those corresponding to Frobenius structures on orbit spaces of finite Coxeter groups. Moreover we find two additional series of polynomial solutions of which one series is of semi-simple type (massive). This result supports Dubrovin's conjecture if modified appropriately.
\end{abstract}

\section{Introduction}

Recently in the physics literature on two-dimensional topological field theory $[1,3]$ a remarkably and amazingly rich system of partial differential equations appeared. Roughly speaking, this system describes the conditions for a quasi-homogeneous function $F=F(t)$ of the variable $t=\left(t_{1}, \ldots, t_{n}\right)$ such that the third-order derivatives form the structure constants of an associative algebra. This system of equations is known as the WittenDijkgraaf-H Verlinde-E Verlinde (WDVV) system.

In the paper [2] and the review article [3], Dubrovin describes, given any finite Coxeter group, how to determine a polynomial solution of the WDVV system. Furthermore, he shows that the algebras associated to these polynomial solutions satisfy a certain semisimplicity assumption. More generally Dubrovin conjectures that any polynomial solution of the WDVV system with positive degrees, such that the associated algebra is semi-simple (massive), can be obtained in such a way.

In this letter we discuss the case $n=4$. We have determined all polynomial solutions with positive degrees, see the appendix. We recover the five solutions associated to the Coxeter groups $A_{4}, B_{4}, D_{4}, F_{4}$ and $H_{4}$. However, we also find two series of additional polynomial solutions. Only one of these series yields semi-simple algebras. This series corresponds to the direct product of two (irreducible) Coxeter groups. This result supports Dubrovin's conjecture if modified slightly, such that appropriate reducible Coxeter groups are included.

\section{The WDVV equations}

\subsection{Definition}

Our aim is to find functions $F(t)=F\left(t^{1}, \ldots, t^{n}\right)$ such that the third-order derivatives,

$$
c_{\alpha \beta \gamma}(t)=\frac{\partial^{3} F(t)}{\partial t^{\alpha} \partial t^{\beta} \partial^{\gamma}}
$$

obey the following conditions, cf [3]. 
1. Normalization

$$
c_{1 \alpha \beta}= \begin{cases}0 & \text { if } \alpha+\beta \neq n+1 \\ 1 & \text { if } \alpha+\beta=n+1 .\end{cases}
$$

We introduce the metric $\eta^{\alpha \beta}=\eta_{\alpha \beta}=c_{1 \alpha \beta}$.

2. Associativity

The functions

$$
c_{\alpha \beta}^{\gamma}(t)=\sum_{\epsilon} \eta^{\gamma \epsilon} c_{\epsilon \alpha \beta}
$$

for any $t$, must define an $n$-dimensional associative algebra with basis $e_{1}, \ldots, e_{n}$ and product given by

$$
e_{\alpha} \cdot e_{\beta}=\sum_{\gamma} c_{\alpha \beta}^{\gamma} e_{\gamma}
$$

\section{Homogeneity}

$F(t)$ must be quasihomogeneous in the variables $t^{1}, \ldots, t^{n}$, i.e. there must exist constants $d_{1}, d_{2}, \ldots, d_{n}$ and $d_{F}$ such that

$$
\sum_{\alpha} d_{\alpha} t^{\alpha} \frac{\partial F}{\partial t^{\alpha}}=d_{F} F
$$

This system of conditions, we call, following [3], the WDVV equations [1,4]. We assumed that the metric $\eta_{\alpha \beta}$ is in standard form. We will restrict our attention to the case that $d_{1}, \ldots, d_{n}$ are all strictly positive. In this case we will assume (unless stated differently) that $d_{1}=1$, and we will write, following physical conventions, $d_{F}=3-d$. For physical reasons we will also assume that $d>0$.

The associativity condition leads to an overdetermined system of partial differential equations (PDEs),

$$
\sum_{\lambda} \frac{\partial^{3} F(t)}{\partial t^{\alpha} \partial t^{\beta} \partial^{\lambda}} \cdot \frac{\partial^{3} F(t)}{\partial t^{\gamma} \partial t^{\delta} \partial^{n+1-\lambda}}=\sum_{\lambda} \frac{\partial^{3} F(t)}{\partial t^{\gamma} \partial t^{\beta} \partial^{\lambda}} \cdot \frac{\partial^{3} F(t)}{\partial t^{\alpha} \partial t^{\delta} \partial^{n+1-\lambda}}
$$

for any $\alpha, \beta, \gamma$ and $\delta$. From these equations, the dependency of $F$ on $t^{1}$ can be solved completely. Namely we get

$$
F(t)=\frac{1}{2} t^{1} \sum_{\alpha=2}^{n} t^{\alpha} t^{n-\alpha+1}+f\left(t^{2}, \ldots, t^{n}\right) .
$$

Now for $F$ to be homogeneous we need (assuming $d_{1}=1$ and $d_{F}=3-d$ ) that

$$
d_{n}=1-d \quad \text { and } \quad d_{\alpha}+d_{n+1-\alpha}=2-d .
$$

Since we require $d_{n}>0$, we need that $d<1$. Hence $0<d<1$.

\section{Polynomial solutions for $n=4$}

\subsection{The results}

We will denote $t^{2}, t^{3}, t^{4}$ by $x, y, z$. The system (2.2), taking into account (2.3), is now equivalent to

$$
\begin{aligned}
& f_{z z z}=f_{x y z}^{2}-f_{x x y} f_{y z z}-f_{x y y} f_{x z z}+f_{x x z} f_{y y z} \\
& f_{x z z}=-f_{x y y} f_{x x z}+f_{x x x} f_{y y z} \\
& f_{y z z}=-f_{x x y} f_{y y z}+f_{y y y} f_{x x z} \\
& f_{x y z}=-\frac{1}{2} f_{x y y} f_{x x y}+\frac{1}{2} f_{x x x} f_{y y y} .
\end{aligned}
$$


We have determined all polynomial solutions corresponding to the case $d_{1}=1, d_{2} \geqslant d_{3}>0$, $d_{4}>0$ and $d>0$. This was done using the computer algebra package REDUCE [5]. The technique that Dubrovin uses for $n=3$ does not work for $n=4$. However, one can deduce from the conditions on $d_{1}, d_{2}, d_{3}, d_{4}$ and $d$ that $f$ is at most cubic in $x$. This splits the system (3.1)-(3.4) into a system of 18 PDEs, now in two variables $y$ and $z$. Carefully analysing this system leads to the seven solutions presented in the appendix.

\subsection{Discussion of the results}

Studying the seven classes of solutions, we find that the first five correspond to Coxeter groups. For (A.6) it turn out that for generic $c, t$ and $q$ there are no nilpotent elements. For (A.7) the situation is completely different. In this case we have that $e_{2}^{2}=c_{21}\left(2 e_{2}-c_{21} e_{1}\right)$, independent of $t$ and $q$. Now taking $a=c_{21} e_{1}-e_{2}$, we have that $a^{2}=0$. Hence we disregard (A.7).

The solution (A.6) can be interpreted in the following way. In the case $n=2$ there is one series of polynomial solutions

$$
F\left(t^{1}, t^{2}\right)=\frac{1}{2}\left(t^{1}\right)^{2} t^{2}+\alpha\left(t^{2}\right)^{k} .
$$

Now we can take the sum $F\left(t^{1}, t^{2}\right)+F\left(t^{3}, t^{4}\right)$ (with two different $\alpha$ ). This will be a solution of the associativity condition; this construction corresponds to taking the direct sum of the algebras, cf [3]. The unit will now correspond to $t^{1}+t^{3}$. Since $d_{2}$ and $d_{4}$ are the same, $F$ will again be quasi-homogeneous.

Concluding, we can say that in the case $n=4$ all semi-simple solutions correspond to (irreducible) Coxeter groups, or to direct products of compatible Coxeter groups.

In general, one sees that to direct products of Coxeter groups with the same Coxeter numbers, one can associate solutions of the WDVV system. Hence Dubrovin's conjecture should be modified to include these cases.

\section{Appendix}

The weights are rescaled to obtain $d_{4}=2$.

$d=3, d_{1}=5, d_{2}=4, d_{3}=3, d_{4}=2$; corresponds to $A_{4}$ :

$$
\begin{aligned}
& f=c_{30} x^{3}+108 c_{30}^{2} c_{4} x^{2} z^{2}+36 c_{30} c_{4} x y^{2} z+c_{4} y^{4}+864 c_{30}^{2} c_{4}^{2} y^{2} z^{3}+\frac{93312}{5} c_{30}^{4} c_{4}^{3} z^{6} \\
& d=6, d_{1}=8, d_{2}=6, d_{3}=4, d_{4}=2 \text {; corresponds to } B_{4} \text { : } \\
& f=c_{30} x^{3}+9 c_{30} c_{13} x^{2} y z+54 c_{30}^{2} c_{13}^{2} x^{2} z^{3}+c_{13} x y^{3}+27 c_{30} c_{13}^{2} x y^{2} z^{2}+\frac{9}{4} c_{13}^{2} y^{4} z \\
& +27 c_{30} c_{13}^{3} y^{3} z^{3}+\frac{1458}{5} c_{30}^{2} c_{13}^{4} y^{2} z^{5}+\frac{13122}{7} c_{30}^{4} c_{13}^{6} z^{9} \\
& d=4, d_{1}=6, d_{2}=4, d_{3}=4, d_{4}=2 \text { : corresponds to } D_{4} \text { : } \\
& f=c_{30} x^{3} z+c_{11} x y z^{3}+\frac{1}{6} \frac{c_{11}}{c_{30}} y^{3} z+\frac{3}{70} c_{11}^{2} z^{7} \\
& d=10, d_{1}=12, d_{2}=8, d_{3}=6, d_{4}=2 \text {; corresponds to } F_{4} \text { : } \\
& f=c_{30} x^{3} z+\frac{36}{5} c_{30}^{2} c_{4} x^{2} z^{5}+12 c_{30} c_{4} x y^{2} z^{3}+c_{4} y^{4} z+\frac{144}{7} c_{30}^{2} c_{4}^{2} y^{2} z^{7}+\frac{1728}{143} c_{30}^{4} c_{4}^{3} z^{13} \\
& d=28, d_{1}=30, d_{2}=20, d_{3}=12, d_{4}=2 \text {; corresponds to } H_{4} \text { : } \\
& f=c_{30} x^{3} z+\frac{9}{5} c_{30} c_{13} x^{2} y z^{5}+\frac{72}{275} c_{30}^{2} c_{13}^{2} x^{2} z^{11}+c_{13} x y^{3} z^{3}+\frac{3}{5} c_{30} c_{13}^{2} x y^{2} z^{9}+\frac{1}{20} c_{13} y_{30}^{5} z \\
& +\frac{3}{10} c_{13}^{2} y^{4} z^{7}+\frac{3}{25} c_{30} c_{13}^{3} y^{3} z^{13}+\frac{72}{2375} c_{30}^{2} c_{13}^{4} y^{2} z^{19}+\frac{3456}{14046875} c_{30}^{4} c_{13}^{6} z^{31}
\end{aligned}
$$


$d=q-3, d_{1}=q-1, d_{2}=q-1, d_{3}=2, d_{4}=2 ; q \geqslant 4:$

$f=\left(c_{21} y+c_{20} z\right) x^{2}+c_{1}\left(y+\left(-c_{21}+D\right) z\right)^{q}+c_{2}\left(y+\left(-c_{21}-D\right) z\right)^{q}$

with $D=\sqrt{c_{21}^{2}+2 c_{20}}$

$d=2 q-4, d_{1}=2 q-2, d_{2}=2 q-2, d_{3}=2, d_{4}=2 ; q \geqslant 3$ :

$f=c_{21}\left(y-\frac{1}{2} c_{21} z\right) x^{2}+c_{11}\left(y-c_{21} z\right)^{q} x+\left(c_{1} y+c_{2} z\right)\left(y-c_{21} z\right)^{2 q-2}$

\section{References}

[1] Dijkgraaf R, Verlinde H and Verlinde E 1991 Notes on topological string theory and 2D quantum gravity Nucl. Phys. B 35259

[2] Dubrovin B 1993 Differential geometry of the space of orbits of a Coxeter group Preprint SISSA-29/93/FM

[3] Dubrovin B 1994 Geometry of 2D topological field theories Preprint SISSA-89/94/FM

Dubrovin B 1996 Integrable Systems and Quantum Groups (Springer Lecture Notes in Mathematics 1620) (New York: Springer) p 120-348

[4] Witten E 1990 On the structure of the topological phase of two-dimensional gravity Nucl. Phys. B 340 281-332

[5] Hearn A 1995 REDUCE User's Manual, version 3.6 Rand Corporation 